\title{
ESTIMATES FOR A REMAINDER TERM ASSOCIATED WITH THE SUM OF DIGITS FUNCTION
}

\author{
by D. M. E. FOSTER
}

(Received 20 November, 1985)

1. Introduction. If $q(\geqslant 2)$ is a fixed integer it is well known that every positive integer $k$ may be expressed uniquely in the form

$$
k=\sum_{r=0}^{\infty} a_{r}(q, k) q^{r} \text { where } a_{r}(q, k) \in\{0,1, \ldots, q-1\} .
$$

We introduce the 'sum of digits' function

$$
\alpha(q, k)=\sum_{r=0}^{\infty} a_{r}(q, k) .
$$

Both the above sums are of course finite. Although the behaviour of $\alpha(q, k)$ is somewhat erratic, its average behaviour is more regular and has been widely studied.

For an integer $n>1$, let $A(q, n)=\sum_{k=1}^{n-1} \alpha(q, k)$, and define $A(q, 1)=0$. In the particular case when $n=q^{s}(s \geqslant 0)$ it is not difficult to prove that

$$
A\left(q, q^{s}\right)=\frac{1}{2}(q-1) s q^{s},
$$

which suggests the asymptotic result

$$
A(q, n) \sim \frac{\frac{1}{2}(q-1)}{\log q} n \log n \quad \text { as } n \rightarrow \infty .
$$

This was proved in 1940 by Bush [2], and in 1949 Mirsky [7] showed in addition that the error term is $O(n)$ but not $O(n)$, thereby improving a contemporary estimate of Bellman and Shapiro [1]. In 1952, Drazin and Griffiths [4] considered the more general problem of the average of

$$
\alpha_{t}(q, k)=\sum_{r=0}^{\infty}\left\{a_{r}(q, k)\right\}^{t}, \text { where } t \in \mathbb{N} .
$$

They obtained the main term and also gave bounds for the remainder term which are all precise in one direction, and in both directions when $t=1$ and $q=2$ or 3. In particular, for the case $q=2$ they proved that

$$
-\frac{\log (4 / 3)}{\log 2}<\left\{A(2, n)-\frac{n \log n}{2 \log 2}\right\} /(n / 2) \leqslant 0 .
$$

Equality holds on the right when $n=2^{s}$. Also if $n=n(s)$ is of either of the forms

$$
1+2^{2}+2^{4}+\ldots+2^{2 s} \text { or } 2\left(1+2^{2}+2^{4}+\ldots+2^{2 s}\right)
$$

Glasgow Math. J. 29 (1987) 109-129. 
then the

$$
\lim _{s \rightarrow \infty}\left\{A(2, n)-\frac{n \log n}{2 \log 2}\right\} /(n / 2)=-\frac{\log (4 / 3)}{\log 2},
$$

ensuring that the above left hand inequality is best possible. These estimates have also been obtained by McIlroy [6] and Shiokawa [8].

In more recent times, there has been a great deal of work on generalisations of this and related problems, some probabilistic in nature. A paper of Stolarsky [9] in 1977, concerned with digital sums (the case $q=2$ ), contains a brief survey of the history of the problem and, very helpfully, cites sixty two references including the ones already mentioned.

In 1975, Delange [3] obtained a very elegant analytical form for the remainder term, involving a function which is continuous, nowhere differentiable and periodic with period 1 , thereby generalizing an earlier result concerned with the case $q=2$ of Trollope [10]. The case of Cantor representations of integers was also considered by Trollope [11] and more recently by Kirschenhofer and Tichy [5]. Their investigation reduces to a study of

$$
S(q, n)=\left\{A(q, n)-\frac{1}{2}(q-1)\left[\frac{\log n}{\log q}\right] n\right\} / \frac{1}{2}
$$

in the special case when the Cantor representation of an integer $k$ becomes a

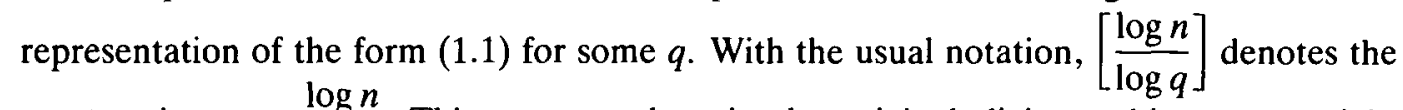
greatest integer $\leqslant \frac{\log n}{\log q}$. This suggests that, in the original digits problem, one might consider directly an estimate for $\frac{S(q, n)}{n}$ and that is the object of this paper. In particular, we obtain best possible upper and lower bounds when $q=2$ and 3 . It is planned to consider later the cases $q=4$ and 5 .

THEOREM 1. If $n \in \mathbb{N}$,

$$
-\frac{2}{13}<\frac{S(2, n)}{n}<1
$$

THEOREM 2. If $n \in \mathbb{N}$,

$$
-\frac{2}{7}<\frac{S(3, n)}{n}<2
$$

The method used to prove Theorems 1 and 2 involves expressing $n$ in the special form $n_{m}(m \in \mathbb{N})$, to be described shortly. Then bounds are obtained for $S\left(q, n_{m}\right) / n_{m}$ in terms of $q$ and $m$, from which Theorems 1 and 2 can be deduced.

Firstly we need to obtain an algebraic expression for $A(q, n)$. If $s \geqslant 2$,

$$
A\left(q, q^{s}\right)=\sum_{1 \leqslant r<q^{s-1}} \alpha(q, r)+\sum_{t=1}^{q-1} \sum_{t q^{s-1} \leqslant r<(t+1) q^{s-1}} \alpha(q, r) .
$$


Putting $r=t q^{s-1}+u$ in the second (inner) sum and using the fact that $\alpha(q, r)=$ $t+\alpha(q, u)$ where $0 \leqslant u<q^{s-1}$, it follows easily that

$$
A\left(q, q^{s}\right)=q A\left(q, q^{s-1}\right)+\frac{1}{2}(q-1) q^{s} .
$$

If $s \geqslant 1$, an inductive proof now yields

$$
A\left(q, q^{s}\right)=\frac{1}{2}(q-1) s q^{s},
$$

and more generally, if $1 \leqslant a<q$,

$$
A\left(q, a q^{s}\right)=a A\left(q, q^{s}\right)+\frac{1}{2} a(a-1) q^{s}
$$

With a slight change of notation, every positive integer $n \not \equiv 0(\bmod q)$ is of the form $n=n_{m}$ where

$$
n_{m}=a_{0} q^{t_{0}}+a_{1} q^{t_{0}+t_{1}}+a_{2} q^{t_{0}+t_{1}+t_{2}}+\ldots+a_{m} q^{t_{0}+t_{1}+t_{2}+\ldots+t_{m}},
$$

for some $m \in \mathbb{N} \cup\{0\}, t_{0}=0$, positive integers $t_{1}, t_{2}, \ldots, t_{m}$ and non-zero coefficients $a_{0}, a_{1}, a_{2}, \ldots, a_{m} \in\{1,2, \ldots, q-1\}$. Given such an integer $n$, for convenience of notation introduce

$$
n_{0}=a_{0} \quad \text { and } n_{i}=a_{0}+a_{1} q^{t_{1}}+\ldots+a_{i} q^{t_{1}+\ldots+t_{i}}
$$

for $1 \leqslant i \leqslant m$. Then

$$
\begin{aligned}
A\left(q, n_{m}\right) & =A\left(q, a_{m} q^{t_{1}+\ldots+t_{m}}\right)+\sum_{a_{m} q^{t_{1}+\ldots+t_{m} \leqslant r<n_{m}}} \alpha(q, r), \\
& =a_{m} A\left(q, q^{t_{1}+\ldots+t_{m}}\right)+\frac{1}{2} a_{m}\left(a_{m}-1\right) q^{t_{1}+\ldots+t_{m}}+a_{m} n_{m-1}+A\left(q, n_{m-1}\right),
\end{aligned}
$$

using (1.7), so that

$$
A\left(q, n_{m}\right)-A\left(q, n_{m-1}\right)=a_{m} A\left(q, q^{t_{1}+\ldots+t_{m}}\right)+a_{m} n_{m-1}+\frac{1}{2} a_{m}\left(a_{m}-1\right) q^{t_{1}+\ldots+t_{m}} .
$$

Iterating this formula and using the fact that $A\left(q, n_{0}\right)=\frac{1}{2} a_{0}\left(a_{0}-1\right)$ we obtain, on addition,

$$
A\left(q, n_{m}\right)-\frac{1}{2} a_{0}\left(a_{0}-1\right)=\sum_{r=1}^{m}\left\{a_{r} A\left(q, q^{t_{1}+\ldots+t_{r}}\right)+a_{r} n_{r-1}\right\}+\frac{1}{2} \sum_{r=1}^{m} a_{r}\left(a_{r}-1\right) q^{t_{1}+\ldots+t_{r}}
$$

However, using (1.6),

$$
\begin{aligned}
& \sum_{r=1}^{m} a_{r} A\left(q, q^{t_{1}+\ldots+t_{r}}\right)=\frac{1}{2}(q-1) \sum_{r=1}^{m} a_{r}\left(t_{1}+\ldots+t_{r}\right) q^{t_{1}+\ldots+t_{r}} \\
& =\frac{1}{2}(q-1) \sum_{r=1}^{m}\left(t_{1}+\ldots+t_{r}\right)\left(n_{r}-n_{r-1}\right)=\frac{1}{2}(q-1) \sum_{r=1}^{m} t_{r}\left(n_{m}-n_{r-1}\right) .
\end{aligned}
$$

$$
\begin{aligned}
A\left(q, n_{m}\right)=\frac{1}{2}(q-1)\left(t_{1}+\ldots+t_{m}\right) n_{m}+\sum_{r=1}^{m}\left(a_{r}-\frac{1}{2}(q-1) t_{r}\right\} n_{r-1} & \\
& +\frac{1}{2} a_{0}\left(a_{0}-1\right)+\frac{1}{2} \sum_{r=1}^{m} a_{r}\left(a_{r}-1\right) q^{t_{1}+\ldots+t_{r}}
\end{aligned}
$$


If $m \in \mathbb{N}$,

$$
q^{t_{1}+\ldots+t_{m}} \leqslant n_{m}<q^{t_{1}+\ldots+t_{m}+1}
$$

so that

$$
t_{1}+\ldots+t_{m}=\left[\frac{\log n_{m}}{\log q}\right]
$$

while if $m=0$,

$$
0=\left[\frac{\log n_{m}}{\log q}\right]
$$

Thus, from (1.3),

$$
S\left(q, n_{m}\right)=\sum_{r=0}^{m} a_{r}\left(a_{r}-1\right) q^{t_{0}+t_{1}+\ldots+t_{r}}+\sum_{r=1}^{m}\left\{2 a_{r}-(q-1) t_{r}\right\} n_{r-1} .
$$

It is easily verified that, if $\beta \in \mathbb{N}$,

$$
\frac{S\left(q, q^{\beta} n_{m}\right)}{q^{\beta} n_{m}}=\frac{S\left(q, n_{m}\right)}{n_{m}}
$$

so that there is no loss of generality in assuming that $n=n_{m}$ is of the form (1.8).

As already mentioned, it is our aim to prove Theorems 1 and 2 in a stronger form, and we now introduce

$$
h_{2}(m)=\frac{2\left(2^{2 m}-1\right)}{13.2^{2 m}-1} \text { and } h_{3}(m)=\frac{6\left(3^{m}-1\right)}{7.3^{m+1}-5} \text { for } m \in \mathbb{N} \cup\{0\}
$$

Each of these functions is monotonic increasing. In fact $h_{2}(1)=0.1176 \ldots, h_{2}(2)=$ $0.1449 \ldots, \quad h_{2}(3)=0.1516 \ldots, \quad h_{2}(4)=0.1532 \ldots \quad$ and, as $m \rightarrow \infty, \quad h_{2}(m) \rightarrow \frac{2}{13}=$ $0.1538 \ldots$ Also $h_{3}(1)=0.2068 \ldots, h_{3}(2)=0.2608 \ldots, h_{3}(3)=0.2775 \ldots$ and, as $m \rightarrow$ $\infty, h_{3}(m) \rightarrow \frac{2}{7}=0.2857 \ldots$

THEOREM $1^{*}$. If $m \geqslant 0$,

$$
-h_{2}(m) \leqslant \frac{S\left(2, n_{m}\right)}{n_{m}} \leqslant 1-\frac{m+1}{2^{m+1}-1} .
$$

Equality holds on the right when $n_{m}=1+2+2^{2}+\ldots+2^{m}$, and on the left when $n_{m}=1+2^{2}+2^{4}+\ldots+2^{2(m-1)}+2^{2(m+1)}(m \geqslant 1)$.

THEOREM $2^{*}$. If $m \geqslant 0$,

$$
-h_{3}(m) \leqslant \frac{S\left(3, n_{m}\right)}{n_{m}} \leqslant 2\left\{1-\frac{m+1}{3^{m+1}-1}\right\} .
$$

Equality holds on the right, when $n_{m}=2\left(1+3+3^{2}+\ldots+3^{m}\right)$, and on the left, for $m \geqslant 3$, when $n_{m}=2+3^{2}+3^{3}+3^{4}+\ldots+3^{m-1}+3^{m}+3^{m+2}$.

For each of these theorems it is the right hand inequality which is the easiest to establish. In fact we can prove a general theorem in this respect. 
THEOREM $3^{*}$. If $q \geqslant 2$ and $m \geqslant 0$,

$$
\frac{S\left(q, n_{m}\right)}{n_{m}} \leqslant(q-1)\left\{1-\frac{m+1}{q^{m+1}-1}\right\}
$$

with equality when $n_{m}=(q-1)\left(1+q+q^{2}+\ldots+q^{m}\right)=q^{m+1}-1$. Since every positive integer is of the form $n_{m}$ or $q^{\beta} n_{m}$ for some $\beta \in \mathbb{N}$, it is clear that the starred theorems give rise to Theorems 1 and 2 .

I should like to express my thanks to my colleague Dr G. M. Phillips for computational work which enabled the value of $h_{3}(m)$, upon which the proof of Theorem $2^{*}$ depends, to be determined for small values of $m$. I should also like to thank the referee for some very helpful comments.

2. The proofs of each of Theorems $1^{*}-3^{*}$ are inductive starting with the small values of $m$. The following identities, derived from (1.10), will be of later use.

$$
\begin{array}{rr}
S\left(q, n_{m}\right)=S\left(q, n_{m-1}\right)+a_{m}\left(a_{m}-1\right) q^{t_{1}+\ldots+t_{m}}+\left\{2 a_{m}-(q-1) t_{m}\right\} n_{m-1} & (m \geqslant 1) ; \\
S\left(q, n_{m}\right)=a_{0}\left(a_{0}-1\right)+a_{0} \sum_{r=1}^{m}\left\{2 a_{r}-(q-1) t_{r}\right\} & \\
+q^{t_{1}} S\left(q, a_{1}+a_{2} q^{t_{2}}+a_{3} q^{t_{2}+t_{3}}+\ldots+a_{m} q^{t_{2}+t_{3}+\ldots+t_{m}}\right) & (m \geqslant 1) ;
\end{array}
$$

and, for any integer $l$, with $2 \leqslant l \leqslant m-1$,

$$
\begin{aligned}
S\left(2, n_{m}\right)= & \sum_{j=1}^{l}\left\{2^{t_{0}+t_{1}+\ldots+t_{j-1}} \sum_{r=j}^{m}\left(2-t_{r}\right)\right\} \\
& +2^{t_{1}+\ldots+t_{i-1}+t_{l}} S\left(2,1+2^{t_{+1}}+2^{t_{+1}+t_{i+2}}+\ldots+2^{t_{+1}+t_{+2}+\ldots+t_{m}}\right)
\end{aligned}
$$

where $t_{0}=0$, as before.

Lemma. For $m \geqslant 1$ and $n_{m}$ as in (1.8) and (1.9),

$$
\frac{n_{m-1}}{n_{m}}<\frac{1}{1+a_{m} q^{t_{m}-1}}
$$

Proof. For $0 \leqslant i \leqslant m-1$ we have $0<a_{i} \leqslant q-1$, giving

$$
\begin{aligned}
& n_{m-1} \leqslant(q-1)\left(1+q^{t_{1}}+q^{t_{1}+t_{2}}+\ldots+q^{t_{1}+t_{2}+\ldots+t_{m-1}}\right), \\
& \quad \leqslant(q-1)\left[\frac{q^{t_{1}+\ldots+t_{m-1}+1}-1}{q-1}\right]<q^{t_{1}+\ldots+t_{m-1}+1} .
\end{aligned}
$$

Hence

$$
\begin{aligned}
\frac{n_{m-1}}{n_{m}}= & \frac{n_{m-1}}{n_{m-1}+a_{m} q^{t_{1}+\ldots+t_{m}}}, \\
& <\frac{q^{t_{1}+\ldots+t_{m-1}+1}}{q^{t_{1}+\ldots+t_{m-1}+1}+a_{m} q^{t_{1}+\ldots+t_{m}}}, \\
= & \frac{1}{1+a_{m} q^{t_{m}-1}} .
\end{aligned}
$$


3. Proof of Theorem $3^{*}$. If $m=0$, we have $n_{m}=n_{0}=a_{0}$ so that

$$
\frac{S\left(q, n_{0}\right)}{n_{0}}=\frac{a_{0}\left(a_{0}-1\right)}{a_{0}}=a_{0}-1 \leqslant q-2=(q-1)\left\{1-\frac{1}{q-1}\right\},
$$

which is the result stated.

Now choose $m \geqslant 1$ and assume that

$$
\frac{S\left(q, n_{m-1}\right)}{n_{m-1}} \leqslant(q-1)\left\{1-\frac{m}{q^{m}-1}\right\} .
$$

By (2.1),

$$
\begin{aligned}
S\left(q, n_{m}\right) & =S\left(q, n_{m-1}\right)+a_{m}\left(a_{m}-1\right) q^{t_{1}+\ldots+t_{m}}+\left\{2 a_{m}-(q-1) t_{m}\right\} n_{m-1}, \\
& \leqslant(q-1)\left\{1-\frac{m}{q^{m}-1}\right\} n_{m-1}+\left(a_{m}-1\right)\left(n_{m}-n_{m-1}\right)+\left\{2 a_{m}-(q-1) t_{m}\right\} n_{m-1}, \\
& =\left(a_{m}-1\right) n_{m}+\left\{a_{m}+q-(q-1) t_{m}-\frac{m(q-1)}{q^{m}-1}\right\} n_{m-1}, \\
& \leqslant\left(a_{m}-1\right) n_{m}+\left\{a_{m}+1-\frac{m(q-1)}{q^{m}-1}\right\} n_{m-1}, \quad \text { since } t_{m} \geqslant 1 .
\end{aligned}
$$

Using (2.4) we see that

$$
\begin{aligned}
\frac{S\left(q, n_{m}\right)}{n_{m}} & \leqslant a_{m}-1+\left\{a_{m}+1-\frac{m(q-1)}{q^{m}-1}\right\} \frac{1}{1+a_{m}}, \\
& =a_{m}-\frac{m(q-1)}{\left(q^{m}-1\right)\left(1+a_{m}\right)} .
\end{aligned}
$$

If $1 \leqslant a_{m} \leqslant q-2$ we have

$$
\begin{aligned}
\frac{S\left(q, n_{m}\right)}{n_{m}} & \leqslant q-2-\frac{m}{q^{m}-1} \\
& =(q-1)\left\{1-\frac{m+1}{q^{m+1}-1}\right\}-\left\{1+\frac{m}{q^{m}-1}-\frac{(q-1)(m+1)}{q^{m+1}-1}\right\} .
\end{aligned}
$$

As $\frac{(q-1)(m+1)}{q^{m+1}-1}<1,(1.13)$ follows in this case.

Now suppose that $a_{m}=q-1$ and $t_{m} \geqslant 2$. From (3.2),

$$
\frac{S\left(q, n_{m}\right)}{n_{m}} \leqslant q-2+\left\{1-\frac{m(q-1)}{q^{m}-1}\right\} \frac{n_{m-1}}{n_{m}} .
$$

By (2.4),

$$
\frac{n_{m-1}}{n_{m}} \leqslant \frac{1}{1+(q-1) q}<\frac{1}{(q-1) q} .
$$


Thus

$$
\frac{S\left(q, n_{m}\right)}{n_{m}} \leqslant q-2+\frac{1}{(q-1) q}-\frac{m}{q\left(q^{m}-1\right)} .
$$

It follows that (1.13) will hold if we can prove that

$$
q-2+\frac{1}{(q-1) q}-\frac{m}{q\left(q^{m}-1\right)} \leqslant q-1-\frac{(q-1)(m+1)}{q^{m+1}-1}
$$

or equivalently

$$
\frac{(q-1)(m+1)}{q^{m+1}-1}-\frac{m}{q\left(q^{m}-1\right)} \leqslant \frac{q(q-1)-1}{q(q-1)} .
$$

Since $q^{m+1}-1>q\left(q^{m}-1\right)$ we have

$$
\begin{aligned}
\frac{(q-1)(m+1)}{q^{m+1}-1}-\frac{m}{q\left(q^{m}-1\right)} & <\frac{(q-1)(m+1)-m}{q\left(q^{m}-1\right)}<\frac{(q-1)(m+1)-m}{m(q-1) q} \\
& \leqslant \frac{q(q-1)-1}{q(q-1)} \text { since } \frac{m+1}{m} \leqslant q
\end{aligned}
$$

Hence (3.3) holds. that

Thus we are left with the case $a_{m}=q-1$ and $t_{m}=1$. If $m=1$, it follows from (3.2)

$$
\frac{S\left(q, n_{1}\right)}{n_{1}} \leqslant q-2+(q-1) \frac{n_{0}}{n_{1}}
$$

where

$$
\frac{n_{0}}{n_{1}}=\frac{a_{0}}{a_{0}+(q-1) q} \leqslant \frac{q-1}{q-1+(q-1) q}=\frac{1}{1+q}
$$

Hence

$$
\frac{S\left(q, n_{1}\right)}{n_{1}} \leqslant q-2+\frac{q-1}{q+1}=(q-1)\left\{1-\frac{2}{q^{2}-1}\right\}
$$

as required.

Suppose now that for some integer $l$ with $2 \leqslant l \leqslant m$,

$$
t_{m}=t_{m-1}=\ldots=t_{l}=1, \quad t_{l-1} \geqslant 2 \text { and } a_{m}=q-1 .
$$

We shall now prove that (1.13) holds. To do this requires an improved upper bound for $n_{m-1} / n_{m}$. For, putting $a_{m}=q-1$ and $t_{m}=1$ in (3.2) we see that

$$
\frac{S\left(q, n_{m}\right)}{n_{m}} \leqslant q-2+\left\{q-\frac{m(q-1)}{q^{m}-1}\right\} \frac{n_{m-1}}{n_{m}}
$$


and (1.13) will follow if we can prove that

$$
q-2+\left\{q-\frac{m(q-1)}{q^{m}-1}\right\} \frac{n_{m-1}}{n_{m}} \leqslant(q-1)\left\{1-\frac{m+1}{q^{m+1}-1}\right\} .
$$

On rearranging, this inequality reduces to

$$
\frac{n_{m-1}}{n_{m}} \leqslant \frac{q^{m}-1}{q^{m+1}-1}
$$

To establish (3.6) we make use of (3.4). We have

$$
\begin{aligned}
n_{m-1} \leqslant & (q-1)\left\{1+q^{t_{1}}+q^{t_{1}+t_{2}}+\ldots+q^{t_{1}+t_{2}+\ldots+t_{l-1}}\right\} \\
& +(q-1) q^{t_{1}+\ldots+t_{l-1}+1}\left(1+q+q^{2}+\ldots+q^{m-l-1}\right), \\
\leqslant & (q-1)\left\{\frac{q^{t_{1}+\ldots+t_{l-1}+1}-1}{q-1}-q^{t_{1}+\ldots+t_{l-1}-1}\right\} \\
& +(q-1) q^{t_{1}+\ldots+t_{l-1}+1} \frac{\left(q^{m-t}-1\right)}{q-1} \\
= & q^{t_{1}+\ldots+t_{l-1}-1}\left(q^{m-l+2}-q+1\right)-1, \\
& <q^{t_{1}+\ldots+t_{l-1}-1}\left(q^{m-l+2}-q+1\right) .
\end{aligned}
$$

Hence

$$
\begin{aligned}
\frac{n_{m-1}}{n_{m}}= & \frac{n_{m-1}}{n_{m-1}+a_{m} q^{t_{1}+\ldots+t_{m}}}, \\
& <\frac{q^{t_{1}+\ldots+t_{l-1}-1}\left(q^{m-l+2}-q+1\right)}{q^{t_{1}+\ldots+t_{l-1}-1}\left(q^{m-l+2}-q+1\right)+(q-1) q^{t_{1}+\ldots+t_{l-1}+m-l+1}} \\
= & \frac{q^{m-l+2}-q+1}{q^{m-l+3}-q+1} .
\end{aligned}
$$

Thus (3.5) will follow provided that

$$
\frac{q^{m-l+2}-q+1}{q^{m-l+3}-q+1} \leqslant \frac{q^{m}-1}{q^{m+1}-1}
$$

or equivalently, on rearranging,

$$
q^{m-l+2}\left\{q^{l-2}(q-1)-1\right\} \geqslant 0,
$$

and this is clearly true for $q \geqslant 2$ and $2 \leqslant l \leqslant m$.

Thus (1.13) is now established except possibly when

$$
m \geqslant 2, \quad a_{m}=q-1 \text { and } t_{1}=t_{2}=\ldots=t_{m}=1 .
$$

However in this case (3.5) holds and (3.6) is easily verified, using the fact that $n_{m-1} \leqslant q^{m}-1$. 
4. Proof of Theorem $1 *$. For the case $q=2$, it suffices to prove that, if $n_{m}=2^{t_{0}}+2^{t_{0}+t_{1}}+\ldots+2^{t_{0}+t_{1}+\ldots+t_{m}}$, where $t_{0}=0, t_{1}, \ldots, t_{m} \in \mathbb{N}$ and $m \geqslant 0$ then

$$
\frac{S\left(2, n_{m}\right)}{n_{m}} \geqslant-h_{2}(m) \text { where } h_{2}(m)=\frac{2\left(2^{2 m}-1\right)}{13 \cdot 2^{2 m}-1} \text {. }
$$

When $m=0$, it is clear from (1.10) that $S\left(2, n_{m}\right)=S\left(2, n_{0}\right)=0=-h_{2}(0)$. Thus we can assume that $m \geqslant 1$. As before, introduce

$$
n_{0}=1 \text { and } n_{i}=1+2^{t_{1}}+2^{t_{1}+t_{2}}+\ldots+2^{t_{1}+t_{2}+\ldots+t_{i}} \quad(1 \leqslant i \leqslant m) .
$$

Then (1.10) takes the simple form

$$
S\left(2, n_{m}\right)=\sum_{r=1}^{m}\left(2-t_{r}\right) n_{r-1} .
$$

If $m=1$,

$$
\frac{S\left(2, n_{1}\right)}{n_{1}}=\frac{2-t_{1}}{1+2^{t_{1}}},
$$

and it is easily verified that for $t \in \mathbb{N}$ this function takes a minimum value namely $-\frac{2}{17}=-h_{2}(1)$, when $t_{1}=4$.

Now choose $m \geqslant 2$ and assume that for all integers $m^{\prime}$ satisfying $1 \leqslant m^{\prime} \leqslant m-1$,

$$
\frac{S\left(2, n_{m^{\prime}}\right)}{n_{m^{\prime}}} \geqslant-h_{2}\left(m^{\prime}\right)>-\frac{2}{13} \text {. }
$$

Using (2.1) with $q=2$ and $a_{m}=1$, we have

$$
S\left(2, n_{m}\right)=S\left(2, n_{m-1}\right)+\left(2-t_{m}\right) n_{m-1},
$$

giving

$$
\frac{S\left(2, n_{m}\right)}{n_{m}}=\left\{\frac{S\left(2, n_{m-1}\right)}{n_{m-1}}+2-t_{m}\right\} \frac{n_{m-1}}{n_{m}} .
$$

By the inductive hypothesis and Theorem $3^{*}$,

$$
-\frac{2}{13}<\frac{S\left(2, n_{m-1}\right)}{n_{m-1}}<1 \text {, }
$$

and, by (2.4),

$$
\frac{n_{m-1}}{n_{m}}<\frac{1}{1+2^{t_{m}-1}} \leqslant \frac{1}{2}
$$

Thus if $t_{m}=1$ we have

$$
\frac{S\left(2, n_{m}\right)}{n_{m}}>0>-h_{2}(m)
$$


and if $t_{m}=2$ we have

If $t_{m} \geqslant 3$,

$$
\frac{S\left(2, n_{m}\right)}{n_{m}}>-\frac{1}{2} h_{2}(m-1)>-h_{2}(m)
$$

$$
\begin{aligned}
\frac{S\left(2, n_{m}\right)}{n_{m}} & >-\left(\frac{2}{13}+t_{m}-2\right) \cdot \frac{1}{1+2^{t_{m}-1}}, \\
& =-\left\{\frac{13 t_{m}-24}{13\left(1+2^{t_{m}-1}\right)}\right\} .
\end{aligned}
$$

The function $\frac{13 t_{m}-24}{13\left(1+2^{t_{m}-1}\right)}$ is monotonic decreasing for $t_{m} \geqslant 6$ with the value $0 \cdot 1258 \ldots$ $\left(<h_{2}(m)\right.$ for $\left.m \geqslant 2\right)$ when $t_{m}=6$. Hence (4.1) follows if $t_{m}=1,2$ or if $t_{m} \geqslant 6$. Assume henceforth that

$$
t_{m}=3,4 \text { or } 5 \text {. }
$$

By (2.2), with $q=2, a_{0}=a_{1}=\ldots=a_{m}=1$, we have

$$
S\left(2, n_{m}\right)=\sum_{r=1}^{m}\left(2-t_{r}\right)+2^{t_{1}} S\left(2,1+2^{t_{2}}+2^{t_{2}+t_{3}}+\ldots+2^{t_{2}+t_{3}+\ldots+t_{m}}\right) .
$$

Applying the induction hypothesis again gives

$$
\begin{aligned}
S\left(2, n_{m}\right) & \geqslant \sum_{r=1}^{m}\left(2-t_{r}\right)-2^{t_{1}} h_{2}(m-1)\left\{1+2^{t_{2}}+2^{t_{2}+t_{3}}+\ldots+2^{t_{2}+\ldots+t_{m}}\right\}, \\
& =\sum_{r=1}^{m}\left(2-t_{r}\right)-h_{2}(m-1)\left\{n_{m}-1\right\} .
\end{aligned}
$$

Thus (4.1) will follow provided that

$$
\sum_{r=1}^{m}\left(2-t_{r}\right)+\left\{h_{2}(m)-h_{2}(m-1)\right\} n_{m}+h_{2}(m-1) \geqslant 0 .
$$

If $s \in \mathbb{N}$,

$$
h_{2}(m)-h_{2}(m-s)=\frac{3\left(2^{2 s}-1\right) 2^{2(m-s)+3}}{\left(13 \cdot 2^{2 m}-1\right)\left(13 \cdot 2^{2(m-s)}-1\right)} .
$$

Hence (4.4) is equivalent to

$$
\sum_{r=1}^{m}\left(2-t_{r}\right)+\frac{9 \cdot 2^{2 m+1}}{\left(13 \cdot 2^{2 m}-1\right)\left(13 \cdot 2^{2(m-1)}-1\right)} n_{m}+h_{2}(m-1) \geqslant 0 .
$$

Clearly (4.6) holds $\forall t_{1}, \ldots, t_{m}>0$ with $\sum_{r=1}^{m}\left(2-t_{r}\right) \geqslant 0$. Thus assume now that

$$
\sum_{r=1}^{m} t_{r}=2 m+1+k \quad \text { where } \quad k \geqslant 0 \text {. }
$$


Condition (4.6) then takes the form

$$
\frac{9.2^{2 m+1}}{\left(13.2^{2 m}-1\right)\left(13 \cdot 2^{2(m-1)}-1\right)} n_{m}+h_{2}(m-1) \geqslant k+1 \text {. }
$$

If $m=2$, we have $n_{m}=1+2^{k+5-t_{2}}+2^{k+5}>1+2^{k+5}$, so that (4.8) will follow provided that

$$
\frac{9.2^{5}\left(1+2^{k+5}\right)}{10,557}+h_{2}(1)>k+1 .
$$

The only integral value of $k \geqslant 0$ for which this inequality does not hold is $k=1$. In this case, from (4.7), $t_{1}+t_{2}=6$. By (4.3), therefore,

$$
\left(t_{1}, t_{2}\right)=(3,3),(2,4) \text { or }(1,5) \text {. }
$$

Then $\frac{S\left(2, n_{2}\right)}{n_{2}}=-0 \cdot 1369 \ldots,-0 \cdot 1449 \ldots\left(=-h_{2}(2)\right)$ or $-0 \cdot 1194 \ldots$ in each of these cases respectively, and the case $m=2$ is proved.

Assume henceforth that $m \geqslant 3$. By (4.7),

$$
n_{m}>2^{2 m+k+1-t_{m}}\left(1+2^{t_{m}}\right) \text {. }
$$

Since $\left(13.2^{2 m}-1\right)\left(13.2^{2(m-1)}-1\right)<169.2^{4 m-2},(4.8)$ will follow if we can prove that

$$
\frac{9 \cdot 2^{2 m+1}}{169 \cdot 2^{4 m-2}} \cdot 2^{2 m+k+1-t_{m}}\left(1+2^{t_{m}}\right)+h_{2}(m-1) \geqslant k+1 \text {, }
$$

that is

$$
\frac{9}{169} \cdot 2^{k+4-t_{m}}\left(1+2^{t_{m}}\right)+h_{2}(m-1) \geqslant k+1 .
$$

It is easily verified that this inequality holds when $t_{m}=3$ for all integers $k \geqslant 0$ and, when $t_{m}=4$ or 5 , it holds for all integers $k \geqslant 0$ except $k=1$. Hence we are left with the cases

$$
m \geqslant 3, \quad t_{m}=4 \text { or } 5 \text { and } \sum_{r=1}^{m} t_{r}=2 m+2 .
$$

Now use identity (2.3), with $l=2$, together with the induction hypothesis on $S\left(2,1+2^{t_{3}}+2^{t_{3}+t_{4}}+\ldots+2^{t_{3}+\ldots+t_{m}}\right)$ to obtain

$$
S\left(2, n_{m}\right) \geqslant \sum_{r=1}^{m}\left(2-t_{r}\right)+2^{t_{1}} \sum_{r=2}^{m}\left(2-t_{r}\right)-h_{2}(m-2)\left\{n_{m}-1-2^{t_{1}}\right\} .
$$

Then (4.1) will follow if we can show that

$$
\sum_{r=1}^{m}\left(2-t_{r}\right)+2^{t_{1}} \sum_{r=2}^{m}\left(2-t_{r}\right)+\left\{h_{2}(m)-h_{2}(m-2)\right\} n_{m}+\left(1+2^{t_{1}}\right) h_{2}(m-2) \geqslant 0 .
$$


Using (4.5) with $s=2$ and (4.9) this inequality is equivalent to

$$
\frac{45.2^{2 m-1}}{\left(13.2^{2 m}-1\right)\left(13.2^{2(m-2)}-1\right)} n_{m}+\left(1+2^{t_{1}}\right) h_{2}(m-2) \geqslant 2+2^{t_{1}}\left(4-t_{1}\right)
$$

If $t_{1} \geqslant 5,2+2^{t_{1}}\left(4-t_{1}\right)<0$ and (4.10) follows easily. Hence, in addition to (4.9), assume that

$$
t_{1}=1,2,3 \text { or } 4 .
$$

If $m=3$, (4.9) and (4.11) are only satisfied when

$$
\left(t_{1}, t_{2}, t_{3}\right)=(1,3,4),(1,2,5),(2,2,4),(2,1,5) \text { and }(3,1,4) \text {. }
$$

The corresponding values of $\frac{S\left(2, n_{3}\right)}{n_{3}}$ are

$-0 \cdot 1454, \ldots,-0 \cdot 1198 \ldots,-0 \cdot 1516 \ldots\left(=-h_{2}(3)\right),-0.1263 \ldots$ and $-0.1494 \ldots$ respectively, thus settling the case $m=3$ of the theorem.

Assume henceforth that $m \geqslant 4$. Clearly

$$
n_{m}>2^{2 m+2-t_{m}}\left(1+2^{t_{m}}\right) \text {. }
$$

Accordingly (4.10) will follow if we can show that

$$
\frac{45.2^{2 m-1}}{169.2^{4 m-4}} \cdot 2^{2 m+2-t_{m}}\left(1+2^{t_{m}}\right)+\left(1+2^{t_{1}}\right) h_{2}(m-2) \geqslant 2+2^{t_{1}}\left(4-t_{1}\right),
$$

that is

$$
\frac{45}{169} \cdot 2^{5-t_{m}}\left(1+2^{t_{m}}\right)+\left(1+2^{t_{1}}\right) h_{2}(m-2) \geqslant 2+2^{t_{1}}\left(4-t_{1}\right) .
$$

Equivalently, this is the condition

$$
2+2^{t_{1}}\left(4-t_{1}\right)-\left(1+2^{t_{1}}\right) h_{2}(m-2) \leqslant\left\{\begin{array}{lll}
9 \cdot 0532 \ldots & \text { if } & t_{m}=4 \\
8 \cdot 7869 \ldots & \text { if } & t_{m}=5
\end{array},\right.
$$

which holds for $t_{1}=1,3$ or 4 . Thus it now remains to consider the cases

$$
m \geqslant 4, \quad t_{1}=2, \quad t_{m}=4 \text { or } 5 \text { and } \sum_{r=1}^{m} t_{r}=2 m+2 .
$$

In the following we choose the maximal integer $l$ satisfying

$$
3 \leqslant l \leqslant m-1 \text { and } t_{1}=t_{2}=\ldots=t_{l-2}=2 .
$$

Using identity (2.3) and the inductive hypothesis for

$$
S\left(2,1+2^{t_{+1}}+\ldots+2^{t_{i+1}+\ldots+t_{m}}\right)
$$


we have

$$
\begin{aligned}
S\left(2, n_{m}\right) \geqslant \sum_{r=1}^{m}\left(2-t_{r}\right)+2^{t_{1}} \sum_{r=2}^{m}\left(2-t_{r}\right) & +\ldots+2^{t_{1}+t_{2}+\ldots+t_{l-1}} \sum_{r=l}^{m}\left(2-t_{r}\right) \\
& -h_{2}(m-l)\left\{n_{m}-1-2^{t_{1}}-2^{t_{1}+t_{2}}-\ldots-2^{t_{1}+t_{2} \ldots+t_{l-1}}\right\} .
\end{aligned}
$$

Hence

$$
S\left(2, n_{m}\right) \geqslant-h_{2}(m) n_{m}
$$

provided that

$$
\begin{aligned}
\sum_{r=1}^{m}\left(2-t_{r}\right)+ & 2^{t_{1}} \sum_{r=2}^{m}\left(2-t_{r}\right)+\ldots+2^{t_{1}+t_{2}+\ldots+t_{l-1}} \sum_{r=l}^{m}\left(2-t_{r}\right) \\
& +\left\{h_{2}(m)-h_{2}(m-l)\right\} n_{m}+\left\{1+2^{t_{1}}+2^{t_{1}+t_{2}}+\ldots+2^{t_{1}+t_{2}+\ldots+t_{l-1}}\right\} h_{2}(m-l) \geqslant 0
\end{aligned}
$$

With (4.5), (4.12) and (4.13), this condition takes the form

$$
\begin{aligned}
-\frac{2}{3}\left(2^{2 l-2}-1\right)+\left(t_{l-1}-4\right) 2^{2 l-4+t_{l-1}} & +\frac{3\left(2^{2 l}-1\right) 2^{2(m-l)+3}}{\left(13.2^{2 m}-1\right)\left(13 \cdot 2^{2(m-l)}-1\right)} n_{m} \\
& +\left\{\frac{1}{3}\left(2^{2 l-2}-1\right)+2^{2 l-4+t_{l-1}}\right\} h_{2}(m-l) \geqslant 0 .
\end{aligned}
$$

If

$$
t_{l-1} \geqslant 5, \quad\left(t_{l-1}-4\right) 2^{2 l-4+t_{l-1}} \geqslant 2^{2 l+1}>\frac{2}{3}\left(2^{2 l-2}-1\right)
$$

and (4.14) follows easily. Thus, in addition to the conditions of (4.13), we can assume that

$$
t_{l-1}=1,2,3 \text { or } 4 \text {. }
$$

Obviously,

$$
n_{m}>2^{2 m+2-t_{m}}\left(1+2^{t_{m}}\right) \geqslant 33 \cdot 2^{2 m-3} \text { for } t_{m}=4 \text { or } 5 .
$$

Thus

$$
\frac{3\left(2^{2 l}-1\right) 2^{2(m-l)+3}}{\left(13 \cdot 2^{2 m}-1\right)\left(13 \cdot 2^{2(m-l)}-1\right)} n_{m}>\frac{99}{169}\left(2^{2 l}-1\right),
$$

and (4.14) is a consequence of

$$
T_{1}\left(l, t_{l-1}\right)+T_{2}(l)+T_{3}\left(l, t_{l-1}\right) h_{2}(m-l)>0
$$

subject to $3 \leqslant l \leqslant m-1$ and $t_{l-1} \in\{1,2,3,4\}$, where

$$
T_{1}\left(l, t_{l-1}\right)=-\frac{2}{3}\left(2^{2 l-2}-1\right)+\left(t_{l-1}-4\right) 2^{2 l-4+t_{l-1}}, \quad T_{2}(l)=\frac{99}{169}\left(2^{2 l}-1\right)
$$

and $T_{3}\left(l, t_{l-1}\right)=\frac{1}{3}\left(2^{2 l-2}-1\right)+2^{2 l-4+t_{l-1}}$.

It is easily verified that

$$
T_{1}(l, 1)+T_{2}(l)=\left(\frac{99}{169}-\frac{13}{24}\right) 2^{2 l}+\frac{41}{507}>0,
$$


and (4.16) follows. Also

$$
\begin{aligned}
T_{1}(l, 3)+T_{2}(l)+T_{3}(l, 3) h_{2}(m-l) & =-\frac{41}{507}\left(2^{2 l}-1\right)+\frac{1}{3}\left(7.2^{2 l-2}-1\right) h_{2}(m-l) \\
& =\frac{7}{3} \cdot 2^{2 l-2}\left\{h_{2}(m-l)-\frac{164}{1183}\right\}+\frac{1}{3}\left\{\frac{41}{169}-h_{2}(m-l)\right\} .
\end{aligned}
$$

If $m-l \geqslant 2, h_{2}(m-l) \geqslant h_{2}(2)>\frac{164}{1183}$ and $\frac{41}{169}>\frac{2}{13}>h_{2}(m-l)$, so that (4.16) follows in this case. If $m-l=1$ and $t_{l-1}=t_{m-2}=3$, the conditions of (4.12) and (4.13) can only be satisfied if $t_{m-1}+t_{m}=5$, whence $\left(t_{m-1}, t_{m}\right)=(1,4)$. Thus

$$
t_{1}=\ldots=t_{m-3}=2, \quad t_{m-2}=3, \quad t_{m-1}=1 \text { and } t_{m}=4,
$$

and it may be verified that, in this case,

$$
-\frac{S\left(2, n_{m}\right)}{n_{m}}=\frac{2\left(2^{2 m}-1\right)}{211.2^{2 m-4}-1}<h_{2}(m)
$$

We have, too

$$
T_{1}(l, 4)+T_{2}(l)=\frac{1}{507}\left(425 \cdot 2^{2 l-1}+41\right)>0
$$

and (4.16) follows again.

Now it remains to consider the case $t_{l-1}=2$. In this case we have $l=m-1$, since $l \leqslant m-2\left(l\right.$ chosen maximal) implies $t_{l-1} \neq 2$. For $l=m-1$ only the following two cases are possible, because of (4.12) and (4.13):

$$
\text { (a) } t_{1}=t_{2}=\ldots=t_{m-2}=2, \quad t_{m}=5 \text { and } t_{m-1}=1
$$

and

$$
\text { (B) } t_{1}=t_{2}=\ldots=t_{m-2}=2, \quad t_{m}=4 \text { and } t_{m-1}=2 .
$$

For case $(\alpha)$ it may be verified that

$$
-\frac{S\left(2, n_{m}\right)}{n_{m}}=\frac{13 \cdot 2^{2 m-3}-2}{101 \cdot 2^{2 m-3}-1}<h_{2}(m),
$$

and, for case $(\beta)$, we have

$$
-\frac{S\left(2, n_{m}\right)}{n_{m}}=h_{2}(m)
$$

giving the critical form for $n_{m}$.

5. Proof of Theorem 2*. For the case $q=3$ we have to prove that, if $n_{m}=$ $a_{0} 3^{t_{0}}+a_{1} 3^{t_{0}+t_{2}}+\ldots+a_{m} 3^{t_{0}+t_{1}+\ldots+t_{m}}$ where $t_{0}=0, t_{1}, \ldots, t_{m} \in \mathbb{N}$ and $a_{0}, a_{1}, \ldots, a_{m} \in$ $\{1,2\}$ then, for $m \geqslant 0$,

$$
\frac{S(3, n)}{n_{m}} \geqslant-h_{3}(m) \quad \text { where } \quad h_{3}(m)=\frac{6\left(3^{m}-1\right)}{7.3^{m+1}-5} .
$$


With the usual notation,

$$
n_{0}=a_{0} \text { and } n_{1}=a_{0}+a_{1} 3^{t_{1}}+a_{2} 3^{t_{1}+t_{2}}+\ldots+a_{i} 3^{t_{1}+t_{2}+\ldots+t_{i}} \quad(1 \leqslant i \leqslant m) \text {, }
$$

and (1.10) takes the form

$$
S\left(3, n_{m}\right)=\sum_{r=0}^{m} a_{r}\left(a_{r}-1\right) 3^{t_{0}+t_{1}+\ldots+t_{r}}+2 \sum_{r=1}^{m}\left(a_{r}-t_{r}\right) n_{r-1} .
$$

If $m=0, S\left(3, n_{0}\right)=a_{0}\left(a_{0}-1\right) \geqslant 0=-h_{3}(0)$ and (5.1) holds with equality when $n_{0}=a_{0}=$ 1.

We prove the case $m=1$ separately, before using an inductive proof for the general case. However it is useful first to obtain two preliminary results.

LEMMA 5.1. If $m \geqslant 0$,

$$
\frac{S\left(3, n_{m}\right)}{n_{m}}>-1
$$

Proof. If $m=0, \frac{S\left(3, n_{0}\right)}{n_{0}}=a_{0}-1 \geqslant 0$. Thus choose $m \geqslant 1$ and assume that

$$
\frac{S\left(3, n_{m^{\prime}}\right)}{n_{m^{\prime}}}>-1 \text { for all integers } m^{\prime} \text { with } 0 \leqslant m^{\prime} \leqslant m-1 \text {. }
$$

By (2.1)

$$
\begin{aligned}
S\left(3, n_{m}\right)= & S\left(3, n_{m-1}\right)+a_{m}\left(a_{m}-1\right) 3^{t_{1}+\ldots+t_{m}}+2\left(a_{m}-t_{m}\right) n_{m-1}, \\
& >-n_{m-1}+2\left(1-t_{m}\right) n_{m-1},
\end{aligned}
$$

using the inductive hypothesis and $a_{m} \geqslant 1$. It follows that

$$
\frac{S\left(3, n_{m}\right)}{n_{m}}>-1
$$

provided that

$$
\frac{n_{m-1}}{n_{m}}<\frac{1}{2 t_{m}-1}
$$

By (2.4),

$$
\frac{n_{m-1}}{n_{m}} \leqslant \frac{1}{1+3^{t_{m}-1}}
$$

and it is easily verified that

$$
\frac{1}{1+3^{t_{m}-1}}<\frac{1}{2 t_{m}-1} \text { for all integers } t_{m} \geqslant 1
$$

LEMMA 5.2. If $m \geqslant 0$ and $a_{m}=2$, then $S\left(3, n_{m}\right)>0$. 
Proof. If $m=0, S\left(3, n_{0}\right)=a_{0}\left(a_{0}-1\right)=2$. Thus assume that $m \geqslant 1$. By (2.1), if $a_{m}=2$ we have

$$
\begin{aligned}
S\left(3, n_{m}\right) & =S\left(3, n_{m-1}\right)+2 \cdot 3^{t_{1}+\ldots+t_{m}}+2\left(2-t_{m}\right) n_{m-1}, \\
& >\left(3-2 t_{m}\right) n_{m-1}+2 \cdot 3^{t_{1}+\ldots+t_{m}}
\end{aligned}
$$

using Lemma 5.1.

Thus if $t_{m}=1, S\left(3, n_{m}\right)>0$. If $t_{m} \geqslant 2$,

$$
S\left(3, n_{m}\right)=2 \cdot 3^{t_{1}+\ldots+t_{m}}-\left(2 t_{m}-3\right) n_{m-1} \text {. }
$$

But $n_{m-1}<3^{t_{1}+\ldots+t_{m-1}+1}$, and it is easily verified that

$$
\left(2 t_{m}-3\right) 3^{t_{1}+\ldots+t_{m-1}+1}<2.3^{t_{1}+\ldots+t_{m}} \text { for } t_{m} \geqslant 2 \text {, }
$$

giving $S\left(3, n_{m}\right)>0$.

Proof of $\frac{S\left(3, n_{1}\right)}{n_{1}} \geqslant-\frac{6}{29}$. We have $n_{1}=a_{0}+a_{1} 3^{t_{1}}$ and $S\left(3, n_{1}\right)=a_{0}\left(a_{0}-1\right)+a_{1}\left(a_{1}-\right.$ 1) $3^{t_{1}}+2\left(a_{1}-t_{1}\right) a_{0}$. By Lemma 5.2, we can assume that $a_{1}=1$.

If $a_{0}=1$, we have

$$
\frac{S\left(3, n_{1}\right)}{n_{1}}=\frac{2\left(1-t_{1}\right)}{1+3^{t_{1}}}
$$

For $t_{1}=1,2$ and $3, \frac{S\left(3, n_{1}\right)}{n_{1}}$ takes the values $0,-\frac{1}{5}$ and $-\frac{1}{7}$ respectively, and thereafter continues to increase towards 0 as $t_{1} \rightarrow \infty$.

If $a_{0}=2$, we have

$$
\frac{S\left(3, n_{1}\right)}{n_{1}}=\frac{2\left(3-2 t_{1}\right)}{2+3^{t_{1}}}
$$

For $t_{1}=1,2$ and $3, \frac{S\left(3, n_{1}\right)}{n_{1}}$ takes the values $\frac{2}{5},-\frac{2}{11}$ and $-\frac{6}{29}$ respectively, and then continues to increase towards 0 as $t_{1} \rightarrow \infty$. Hence

$$
\frac{S\left(3, n_{1}\right)}{n_{1}} \geqslant-\frac{6}{29} \text { with equality only when } n_{1}=2+3^{3} \text {. }
$$

Proof of $\frac{S\left(3, n_{m}\right)}{n_{m}} \geqslant-h_{3}(m), \quad(m \geqslant 2)$. Assume that $\frac{S\left(3, n_{m^{\prime}}\right)}{n_{m^{\prime}}} \geqslant-h_{3}\left(m^{\prime}\right)$ for all integers $m^{\prime}$ satisfying $1 \leqslant m^{\prime} \leqslant m-1$. By Lemma 5.2, we can take $a_{m}=1$ and then, by (2.1), we have

$$
S\left(3, n_{m}\right)=S\left(3, n_{m-1}\right)+2\left(1-t_{m}\right) n_{m-1} \text {. }
$$

If $t_{m}=1$,

$$
\frac{S\left(3, n_{m}\right)}{n_{m}}=\frac{S\left(3, n_{m-1}\right)}{n_{m-1}} \cdot \frac{n_{m-1}}{n_{m}}
$$


and the induction hypothesis, together with (2.4), yields

$$
\frac{S\left(3, n_{m}\right)}{n_{m}} \geqslant-\frac{1}{2} h_{3}(m-1)>-h_{3}(m) .
$$

If $t_{m} \geqslant 2$, we have on applying the induction hypothesis

$$
\begin{aligned}
\frac{S\left(3, n_{m}\right)}{n_{m}} & \geqslant-\left\{h_{3}(m-1)+2\left(t_{m}-1\right)\right\} \frac{n_{m-1}}{n_{m}}, \\
& \geqslant-\frac{\left\{h_{3}(m-1)+2\left(t_{m}-1\right)\right\}}{1+3^{t_{m}-1}} .
\end{aligned}
$$

As $h_{3}(m-1)<\frac{2}{7}$, we have $\frac{S\left(3, n_{m}\right)}{n_{m}}>-f\left(t_{m}\right)$ where $f(t)=\frac{2(t-1)+\frac{2}{7}}{1+3^{t-1}}$. Now $f(2)=\frac{4}{7}$, $f(3)=\frac{3}{7}, f(4)=\frac{11}{49}<h_{3}(2)$ and $f(t)$ continues to decrease as $t$ increases, so that (5.1) follows if $t_{m} \geqslant 4$. Thus we only need consider the cases when $t_{m}=2$ or 3 .

By (2.2), we have

$$
S\left(3, n_{m}\right)=a_{0}\left(a_{0}-1\right)+2 a_{0} \sum_{r=1}^{m}\left(a_{r}-t_{r}\right)+3^{t_{1}} S\left(3, a_{1}+a_{2} 3^{t_{2}}+a_{3} 3^{t_{2}+t_{3}}+\ldots+a_{m} 3^{t_{2}+t_{3}+\ldots+t_{m}}\right),
$$

and applying the induction hypothesis once again we see that

$$
S\left(3, n_{m}\right) \geqslant a_{0}\left(a_{0}-1\right)+2 a_{0} \sum_{r=1}^{m}\left(a_{r}-t_{r}\right)-\left(n_{m}-a_{0}\right) h_{3}(m-1) .
$$

Thus $S\left(3, n_{m}\right) \geqslant-h_{3}(m)$ provided that

$$
a_{0}\left(a_{0}-1\right)+2 a_{0} \sum_{r=1}^{m}\left(a_{r}-t_{r}\right)+\left\{h_{3}(m)-h_{3}(m-1)\right\} n_{m}+a_{0} h_{3}(m-1) \geqslant 0 .
$$

Since $0 \leqslant h_{3}(m-1)<h_{3}(m)$ for $m \geqslant 1$, this inequality is easily satisfied when $\sum_{r=1}^{m}\left(a_{r}-t_{r}\right) \geqslant 0$. Thus suppose henceforth that

$$
a_{m}=1, \quad t_{m}=2 \text { or } 3 \text { and } \sum_{r=1}^{m}\left(a_{r}-t_{r}\right)=-1-k \text { where } k \geqslant 0 .
$$

Then (5.2) takes the form

$$
\left\{h_{3}(m)-h_{3}(m-1)\right\} n_{m}+a_{0} h_{3}(m-1) \geqslant a_{0}\left(3-a_{0}+2 k\right) .
$$

The case $m=2$. We have $h_{3}(m)-h_{3}(m-1)=\frac{6}{23}-\frac{6}{29}=\frac{36}{667}$, and $n_{m}=n_{2} \geqslant 1+3^{t_{1}}+$ $3^{t_{1}+t_{2}}$ where, from (5.3),

$$
t_{1}=\left\{\begin{array}{lll}
a_{1}+k & \text { if } & t_{2}=2 \\
a_{1}+k-1 & \text { if } & t_{2}=3
\end{array}\right.
$$


If $t_{2}=2$, (5.4) will follow provided that

$$
\frac{36}{667}\left(1+3^{a_{1}+k}+3^{a_{1}+k+2}\right)+\frac{6}{29} a_{0} \geqslant a_{0}\left(3-a_{0}+2 k\right) .
$$

For $a_{0}=1$, (5.6) holds except when $k=0$ and $a_{1}=1$. In this case, $n_{2}=1+3+3^{3}$ and $-\frac{S\left(n_{2}\right)}{n_{2}}=\frac{8}{31}<\frac{6}{23}$. For $a_{0}=2,(5.6)$ holds except when $k=1$ and $a_{1}=1$. Then $n_{2}=2+3^{2}+3^{4}$ and $-\frac{S\left(3, n_{2}\right)}{n_{2}}=\frac{6}{23}$, giving rise to the critical case.

If $t_{2}=3$, (5.4) will follow provided that

$$
\frac{36}{667}\left(1+3^{a_{1}+k-1}+3^{a_{1}+k+2}\right)+\frac{6}{29} a_{0} \geqslant a_{0}\left(3-a_{0}+2 k\right) .
$$

For $a_{0}=1$, (5.7) holds except when $k=0$ and $a_{1}=1$. But, from (5.5), this implies that $t_{1}=0$ so that this possibility is excluded. For $a_{0}=2$, (5.7) holds except when $k=0$ or 1 and $a_{1}=1$. From (5.5), $k=0$ and $a_{1}=1$ imply once again that $t_{1}=0$. If $k=1$ and $a_{1}=1$ we have $n_{2}=2+3+3^{4}$ and $-\frac{S\left(n_{2}\right)}{n_{2}}=\frac{9}{43}<\frac{6}{23}$.

The case $m \geqslant 3$. We have

and

$$
h_{3}(m)-h_{3}(m-1)=\frac{64 \cdot 3^{m}}{\left(7 \cdot 3^{m+1}-5\right)\left(7 \cdot 3^{m}-5\right)} \geqslant \frac{64}{49 \cdot 3^{m+1}},
$$

$$
\begin{aligned}
n_{m} & \geqslant 1+3^{t_{1}}+3^{t_{1}+t_{2}}+\ldots+3^{t_{1}+t_{2}+\ldots+t_{m-2}}+\left(1+3^{t_{m}}\right) \cdot 3^{t_{1}+\ldots+t_{m-1}}, \\
& \geqslant \frac{1}{2}\left\{3^{m-1}-1+2\left(1+3^{t_{m}}\right) 3^{t_{1}+\ldots+t_{m-1}}\right\} .
\end{aligned}
$$

Thus

$$
\left\{h_{3}(m)-h_{3}(m-1)\right\} n_{m} \geqslant \frac{32}{441}\left\{2\left(1+3^{t_{m}}\right) 3^{t_{1}+\ldots+t_{m-1}-m+1}+1-\frac{1}{3^{m-1}}\right\},
$$

and (5.4) will hold if we can prove that

$$
\frac{32}{441}\left\{2\left(1+3^{t_{m}}\right) 3^{t_{1}+\ldots+t_{m-1}-m+1}+1-\frac{1}{9}\right\} \geqslant a_{0}\left(3-a_{0}+2 k-\frac{6}{23}\right) .
$$

From (5.3) we have the condition

$$
t_{1}+\ldots+t_{m-1}=\left\{\begin{array}{lll}
a_{1}+\ldots+a_{m-1}+k & \text { if } & t_{m}=2 \\
a_{1}+\ldots+a_{m-1}+k-1 & \text { if } & t_{m}=3 .
\end{array}\right.
$$

Suppose first that

$$
a_{1}+\ldots+a_{m-1}=m-1 \text { or equivalently } a_{1}=\ldots=a_{m-1}=1 .
$$

Then (5.8) is equivalent to

$$
T_{1}\left(a_{0}, k\right) \leqslant\left\{\begin{array}{lll}
T_{2}(k) & \text { if } & t_{m}=2, \\
T_{3}(k) & \text { if } & t_{m}=3,
\end{array}\right.
$$


where

$$
T_{1}\left(a_{0}, k\right)=a_{0}\left(3-a_{0}+2 k-\frac{6}{23}\right), \quad T_{2}(k)=\frac{32}{441}\left(20 \cdot 3^{k}+\frac{8}{9}\right)
$$

and

$$
T_{3}(k)=\frac{32}{441}\left(56 \cdot 3^{k-1}+\frac{8}{9}\right) \text {. }
$$

The small values of $k$ give rise to the following values of $T_{1}, T_{2}$ and $T_{3}$ :

$$
\begin{array}{ll}
T_{1}(1,0)=1 \cdot 73 \ldots & T_{2}(0)=1 \cdot 51 \ldots \\
T_{1}(2,0)=1 \cdot 47 \ldots & T_{3}(0)=1 \cdot 41 \ldots \\
T_{1}(1,1)=3 \cdot 73 \ldots & T_{2}(1)=4 \cdot 41 \ldots \\
T_{1}(2,1)=5 \cdot 47 \ldots & T_{3}(1)=4 \cdot 12 \ldots \\
T_{1}(1,2)=5 \cdot 73 \ldots & T_{2}(2)=13 \cdot 12 \ldots \\
T_{1}(2,2)=9 \cdot 47 \ldots & T_{3}(2)=12.25 \ldots \\
T_{1}(1,3)=7 \cdot 73 \ldots & T_{2}(3)=39 \cdot 24 \ldots \\
T_{1}(2,3)=13 \cdot 47 \ldots & T_{3}(3)=36.63 \ldots
\end{array}
$$

As $k$ increases, the values of $T_{2}(k)$ and $T_{3}(k)$ increase exponentially while those of $T_{1}\left(a_{0}, k\right)$ increase only linearly, and it is not difficult to prove that $T_{1}\left(a_{0}, k\right)<T_{i}(k)$ if $i=2$ or 3 for all $k \geqslant 4$, and (5.11) holds. From inspection of the above table, we see that (5.11) is true except in the following cases:

and

(i)

$$
k=0:\left(a_{0}, t_{m}\right)=(1,2),(1,3) \text { or }(2,3)
$$

(ii)

$$
k=1:\left(a_{0}, t_{m}\right)=(2,2) \text { or }(2,3) .
$$

However, from (5.9) and (5.10), it is not possible to have $t_{m}=3$ when $k=0$ since this would imply that $t_{1}+\ldots+t_{m-1}=m-2$. Thus case (i) reduces to

(i)'

$$
k=0:\left(a_{0}, t_{m}\right)=(1,2) \text {. }
$$

From (5.9) and (5.10), this implies that $\left(a_{0}, a_{1}, \ldots, a_{m}\right)=(1,1, \ldots, 1)$ and $\left(t_{1}, \ldots, t_{m-1}, t_{m}\right)=(1, \ldots, 1,2)$. Hence

$$
n_{m}=1+3+3^{2}+\ldots+3^{m-1}+3^{m+1}=\frac{1}{2}\left(3^{m}-1\right)+3^{m+1}=\frac{1}{2}\left(7.3^{m}-1\right)
$$

and $S\left(3, n_{m}\right)=2(1-2) \cdot \frac{1}{2}\left(3^{m}-1\right)=-\left(3^{m}-1\right)$, giving

$$
-\frac{S\left(3, n_{m}\right)}{n_{m}}=\frac{2\left(3^{m}-1\right)}{7.3^{m}-1}<\frac{6\left(3^{m}-1\right)}{7.3^{m+1}-5}
$$

Now consider case (ii). If $k=1$ and $t_{m}=3$, we see from (5.9) and (5.10) that $t_{1}+\ldots+t_{m-1}=m-1$, giving $t_{1}=\ldots=t_{m-1}=1$. Thus, if $\left(a_{0}, t_{m}\right)=(2,3)$, we have

$$
\left(a_{0}, a_{1}, \ldots, a_{m}\right)=(2,1, \ldots, 1) \text { and }\left(t_{1}, \ldots, t_{m-1}, t_{m}\right)=(1, \ldots, 1,3) \text {. }
$$


This gives

$$
n_{m}=2+3+3^{2}+\ldots+3^{m-1}+3^{m+2}=\frac{1}{2}\left(3^{m}+1\right)+3^{m+2}=\frac{1}{2}\left(19 \cdot 3^{m}+1\right)
$$

and

$$
S\left(3, n_{m}\right)=2+2(1-3) \cdot \frac{1}{2}\left(3^{m}+1\right)=-2 \cdot 3^{m}
$$

Hence

$$
-\frac{S\left(3, n_{m}\right)}{n_{m}}=\frac{4 \cdot 3^{m}}{19 \cdot 3^{m}+1}<\frac{6\left(3^{m}-1\right)}{7 \cdot 3^{m+1}-5} \Leftrightarrow\left(15 \cdot 3^{m}+1\right)\left(3^{m}-3\right)>0
$$

which is true for $m \geqslant 1$.

If $k=1$ and $\left(a_{0}, t_{m}\right)=(2,2)$ we have, from (5.9) and (5.10), $\left(a_{0}, a_{1}, \ldots, a_{m}\right)=$ $(2,1, \ldots, 1)$ and $t_{1}+\ldots+t_{m-1}=m$. If $t_{1}=2$ then $t_{2}=\ldots=t_{m-1}=1$, and we have

$$
n_{m}=2+3^{2}+3^{3}+\ldots+3^{m}+3^{m+2}=\frac{1}{2}\left(3^{m+1}-5\right)+3^{m+2}=\frac{1}{2}\left(7 \cdot 3^{m+1}-5\right)
$$

and

$$
S\left(3, n_{m}\right)=2+2(1-2) \cdot 2+2(1-2) \cdot \frac{1}{2}\left(3^{m+1}-5\right)=-3\left(3^{m}-1\right)
$$

Thus

$$
\frac{S\left(3, n_{m}\right)}{n_{m}}=-\frac{6\left(3^{m}-1\right)}{7.3^{m+1}-5}
$$

and this is the critical case. Alternatively if $t_{1}=1$, there is some $r$ with $2 \leqslant r \leqslant m-1$ such that

$$
t_{1}=\ldots=t_{r-1}=1, t_{r}=2, \quad t_{r+1}=\ldots=t_{m-1}=1 .
$$

[If $r=m-1$, this condition should read $t_{1}=\ldots=t_{r-1}=1, t_{r}=2$.] In this case

$$
n_{m}=2+3+\ldots+3^{r-1}+3^{r+1}+\ldots+3^{m}+3^{m+2},
$$

so that

$$
n_{r-1}=\frac{1}{2}\left(3^{r}+1\right), \quad n_{m-1}=\frac{1}{2}\left(3^{m+1}-2 \cdot 3^{r}+1\right)
$$

and

$$
n_{m}=\frac{1}{2}\left(7 \cdot 3^{m+1}-2 \cdot 3^{r}+1\right) .
$$

Also it may be verified that $S\left(3, n_{m}\right)=-\left(3^{m+1}-3^{r}\right)$, giving

$$
\begin{gathered}
-\frac{S\left(3, n_{m}\right)}{n_{m}}=\frac{6\left(3^{m}-3^{r-1}\right)}{7 \cdot 3^{m+1}-2 \cdot 3^{r}+1} \leqslant \frac{6\left(3^{m}-1\right)}{7 \cdot 3^{m+1}-5} \\
\Leftrightarrow\left(5 \cdot 3^{m+1}+1\right)\left(3^{r-1}-1\right) \geqslant 0
\end{gathered}
$$

which is true. 
It remains to observe that when

$$
a_{1}+\ldots+a_{m-1}=m-1+u \text { where } u \geqslant 1,
$$

the values of $T_{2}(k)$ and $T_{3}(k)$ in (5.11) are replaced by $T_{2}(k+u)$ and $T_{3}(k+u)$ while those of $T_{1}\left(a_{0}, k\right)$ remain unaltered. Inspection of the tabulated values shows that the inequalities (5.11) are always satisfied. Hence the theorem is proved.

\section{REFERENCES}

1. R. Bellman, and H. N. Shapiro, On a problem in additive number theory, Ann. of Math. (2), 49 (1948), 333-40.

2. L. E. Bush, An asymptotic formula for the average sums of the digits of integers, Amer. Math. Monthly 47 (1940), 154-6.

3. H. Delange, Sur la fonction sommatoire de la fonction 'somme des chiffres', Enseignment Math. 21 (1975) 31-47.

4. M. P. Drazin, and J. S. Griffiths, On the decimal representation of integers, Proc. Cambridge Philos. Soc. 48 (1952), 555-565.

5. P. Kirschenhofer, and R. F. Tichy, On the distribution of digits in Cantor representations of integers, J. Number Theory 18 (1984), 121-134.

6. M. D. Mcllroy, The number of 1's in binary integers: bounds and extremal properties, SIAM J. Comput. 3 (1974), 255-261.

7. L. Mirsky, A theorem on representations of integers in the scale of r, Scripta Math. 15 (1949), 11-12. 167-176.

8. I. Shiokawa, On a problem in additive number theory, Math. J. Okayama Univ. 16 (1974),

9. K. B. Stolarsky, Power and exponential sums of digital sums related to binomial coefficient parity, SIAM J. Appl. Math. 32 (1977), 717-730. $21-25$.

10. J. R. Trollope, An explicit expression for binary digital sums, Math. Mag. 41 (1968), 690-694.

11. J. R. Trollope, Generalized bases and digital sums, Amer Math. Monthly 74 (1967),

Mathematical Institute

UNIVERSITY OF ST ANDREWS

NORTH HAUGH

St ANDRews, KY16 9SS 